\title{
Instrumento para evaluar el estado de la gestión de mantenimiento en plantas de bioproductos: Un caso de estudio
}

\author{
Instrument to assess the status of maintenance management on \\ bioproduct plants: Case study
}

\author{
Armando Díaz Concepción ${ }^{1 *} \quad$ Alfredo Del Castillo Serpa ${ }^{2} \quad$ Leisis Villar Ledo $^{3}$
}

Recibido 19 de febrero de 2016, aceptado 29 de junio de 2016

Received: February 19, 2016 Accepted: June 29, 2016

\begin{abstract}
RESUMEN
En el presente trabajo se propone un procedimiento para diseñar un instrumento tipo encuesta para estimar el estado de la gestión del mantenimiento en plantas de bioproductos. Se describe la metodología para su validación, por medio de las pruebas de validez y confiabilidad, obteniéndose un instrumento que permite conocer el estado de mantenimiento en plantas de bioproductos con un 90,8\% de confiabilidad de los datos medidos. La herramienta se aplicó a un caso de estudio como forma de demostrar la pertinencia de la misma. Se obtuvo como resultado que la evaluación de la planta objeto de estudio catalogada de mala exponiendo los renglones que pueden ser objeto de mejora.
\end{abstract}

Palabras clave: Bioproductos, gestión del mantenimiento, validación, confiabilidad.

\section{ABSTRACT}

This paper has the goal to propose a procedure to design an instrument base on interview to assess the state of the management maintenance in the bio-products plants. On describe the methodology to do the validation thought by test of validation and reliability. This instrument, allows knowing with 90,8\% of reliability based on the information the condition of maintenance in this plants. The application in the case study showed the rightness of this instrument. The assessments of this plant produce as a result the bad condition of the management maintenance and the possibility of improvement.

Keywords: Bioproducts, maintenance management, validation, reliability.

\section{INTRODUCCIÓN}

En las últimas décadas la concepción del mantenimiento a nivel internacional ha cambiado, pasando de una actividad reactiva a adoptar una concepción proactiva, dotándolo de una visión de negocio en su expresión más amplia. Este novedoso concepto implica desarrollar una cuidadosa preparación de las acciones a emprender, valiéndose para ello de la organización y desarrollo de la "gestión de mantenimiento" [1].

1 Centro de Estudios de Ingeniería en Mantenimiento. Facultad de Ingeniería Mecánica. Universidad Tecnológica de la Habana "José Antonio Echeverría", CUJAE. La Habana, Cuba. E-mail: adiaz@ ceim.cujae.edu.cu

2 Centro de Estudios de Matemática. Universidad Tecnológica de la Habana “José Antonio Echeverría”, CUJAE. La Habana, Cuba. E-mail: acastillo@cemat.cujae.edu.cu

3 Departamento de Ingeniería Industrial. Facultad de Ingeniería Industrial. Universidad Tecnológica de la Habana "José Antonio Echeverría”, CUJAE. La Habana, Cuba. E-mail: leisis@ind.cujae.edu.cu

* Autor de correspondencia 
En la actualidad, en Cuba, no se ha establecido una política a nivel de país para diagnosticar y evaluar la gestión de mantenimiento, estando controlada por diversos métodos. La principal consecuencia de esta situación es que no se realiza una evaluación homogénea e igualmente confiable de la actividad mantenimiento en diferentes entidades [1]. Es por esto que se hace necesario establecer una política práctica que recoja diferentes aspectos diagnosticables y evaluables de la gestión de mantenimiento en las plantas de producción de productos biológicos bajo criterios de certificación.

La forma de gestionar el mantenimiento está estrechamente vinculada al proceso de producción o servicio que se realice. En Cuba, en consonancia con los criterios mundiales, se experimenta durante los últimos años un desarrollo en la producción de plaguicidas biológicos para el control de plagas [2].

Este nuevo tipo de producción conlleva a la definición de estrategias de mantenimientos contextualizadas a este tipo de proceso. La producción de estos bioproductos se realiza en procesos industriales caracterizados por ser una producción no continua, realizada por lotes, con períodos de obtención de los productos de hasta 14 días; necesidad de contar con una alta disponibilidad de equipamiento sumamente especializado, de alta complejidad y costosos; además existe la necesidad imperativa de garantizar alta seguridad operacional al trabajar con microorganismos vivos, por lo que se trata de plantas sujetas a rigurosos controles ambientales [3].

A partir de estas características, surge la necesidad de contar con una estrategia de mantenimiento que apoye los aspectos anteriores. Para este análisis, es necesario realizar un diagnóstico del estado actual de la gestión de mantenimiento en varias de estas plantas.

Diferentes autores han propuesto instrumentos para determinar el estado de la gestión del mantenimiento, como en el trabajo titulado" Auditoría y Evaluación de la Gestión de la Calidad en el Mantenimiento" [4], que plantea una metodología para estimar el estado de la gestión de mantenimiento en industrias de producción o de servicios. Las tres principales limitaciones de dicha metodología son: primero, la necesidad de la realización de auditorías in situ; segundo, aunque tiene elementos de control sobre la seguridad, los mismos son limitados para estos tipos de planta, donde este control pasa a tomar un carácter de interés principal; y tercero, no presupone la toma de decisiones para la implementación de estrategias referidas a sistemas de mantenimiento propios de estas plantas como son el Mantenimiento Centrado en la Confiabilidad RCM y el Mantenimiento Productivo Total TPM.

En el trabajo del MSc, Emiro Vasquez [5], se expone un caso de estudio que tiene como elemento importante el auditar sobre la base de la seguridad. La metodología está referida a otro campo de investigación no haciéndose factible su implementación para el campo referido a esta investigación. En el mismo trabajo se exponen otros métodos como son MES, MQS, Matriz Cualitativa de Mantenimiento (MCM). En Cuba se pueden relacionar la "Metodología para la evaluación de mantenimiento industrial y eficiencia energética", perteneciente al Ministerio de la Industria Sideromecánica, SIME, pero la misma está referida a entidades propias de este antiguo ministerio, la que está muy limitada en sus análisis de seguridad.

En el trabajo de Liudmila Ruiz [6], se expone otro método para el diagnóstico donde aplica encuestas personalizadas y toma como base la encuesta sobre la calidad del servicio o producto, por lo que se aplica en el trabajo de investigación la parte teórica sobre la satisfacción del cliente pero como este tipo de plantas tiene como base de atención priorizada la seguridad no aplica en su mayoría la misma.

En la actualidad el desarrollo del mantenimiento va enfocado, entre otras directivas, a disminuir costos, aumentar la disponibilidad y confiabilidad operacional, optimizar el rendimiento y aumentar el período de vida útil de los activos [3]. Para lograr estos propósitos se diseñan líneas y pautas a seguir que van desde las estrategias a emprender hasta las medidas organizativas que posibiliten el buen funcionamiento de las directrices tomadas. El objetivo del presente trabajo es diseñar un instrumento que permita evaluar el estado de la gestión de mantenimiento en planta de bioproductos. Para demostrar la idoneidad de la herramienta se implementará la misma en un caso de estudio. 


\section{ANÁLISIS TEÓRICO}

\section{Diseño de un modelo de planta típica, diferentes procesos y áreas}

Para este estudio se consideró como base una planta típica que incluyera las áreas, sistemas y equipos comunes a los distintos tipos de plantas instaladas en el país [3]. A continuación se mencionan las áreas generales de una planta de producción de bioproductos:

1. Área de laboratorio y preparación de inóculo. (Preinóculo).

2. Área de producción. (Producción).

3. Área de recuperación. (Recuperación).

4. Área de disposición del producto final. (Terminación).

5. Área de preparación de materia prima. (Materias primas).

6. Área de servicios. (Aire, vapor, agua y ventilación).

7. Área de tratamiento de residuales. (Residuales).

8. Área civil. (Construcción Civil).

Parámetros necesarios para realizar el diagnóstico de la función mantenimiento

El diagnóstico de la función mantenimiento consiste en el examen y evaluación que se realiza a una entidad para establecer el grado de economía, eficiencia y eficacia en la planificación, control y uso de los recursos y comprobar la observancia de las disposiciones establecidas, con el objetivo de verificar la utilización más racional de los recursos y mejorar las actividades y materias examinadas [1].

La efectividad de la gestión del mantenimiento solo puede ser evaluada y medida por el análisis exhaustivo de una amplia variedad de factores que, en su conjunto, constituyen la aportación del mantenimiento a la calidad de los servicios prestados.

Se define como calidad en la gestión de mantenimiento al grado en que los productos o servicios cumplen con las exigencias de la gente que los utiliza $[1,6]$.

La calidad en la gestión del mantenimiento se enfoca a índices objetivos. En ocasiones, atendiendo a la cantidad de información estadística acumulada, a datos no confiables y a desconocimiento de patrones que denotan un determinado indicador, se hace necesario acudir a utilizar medidas subjetivas o blandas como indicadores de la calidad. Estas medidas son blandas porque se enfocan hacia las percepciones y actitudes, en lugar de hacia criterios más concretos y objetivos. Estas se incluyen en cuestionarios de satisfacción de clientes para determinar el estado de calidad de un sistema [7].

El objeto de estudio de esta investigación fueron las gerencias a las que se da el servicio de mantenimiento.

El primer paso del proceso es identificar los requisitos del cliente, los que constituyen las características más importantes del servicio que se desea brindar. El cumplimiento de estos requerimientos definirá la calidad del servicio. [7].

En el segundo paso, se desarrolla y evalúa el cuestionario cuyo objetivo final es obtener un listado de preguntas que permita la valoración de una información concreta, sobre las percepciones de los clientes. El tercer paso, es la aplicación del cuestionario.

Diseño de una encuesta para analizar el estado de gestión de manteamiento en plantas de producción de bioproductos

Los parámetros del diseño de una encuesta son: variable de investigación o rendimiento, indicadores, dimensiones e ítems [8-9].

1. Variable de investigación o rendimiento: grado de la gestión de mantenimiento en plantas de bioproductos.

2. Indicadores: son las variables que denotarán a la variable de investigación.

3. Dimensiones: Son los niveles en que se expresan las variables indicadoras (ejemplos: Nivel del mantenimiento dentro de la organización, Comunicación entre departamentos, etc).

4. Ítemes: Son las preguntas que servirán para evaluar la dimensión específica.

En este caso y para una mejor estructura de las encuestas se realizaron dos modelos. El Modelo I recoge toda aquella información relacionada con la estructura organizativa, el área específica de mantenimiento, los recursos humanos, producción, economía y logística, las cuáles son de referencias generales o sea relativas a los parámetros independientes de los procesos. El Modelo II recoge lo relacionado con el equipamiento y estrategias de mantenimiento el que está dirigido con mayor incidencia hacia los procesos. 


\section{Validez del instrumento}

La validez está referida al grado en que la evidencia apoya las inferencias o deducciones sacadas de las puntuaciones derivadas de las mediciones, no existiendo estadística alguna que proporcione un índice general de la validez de las inferencias realizadas sobre las puntuaciones. En ella se deben de tener tres tipos de evidencias, relacionadas con el contenido, el criterio y el constructo.

La validez del contenido se ocupa del grado en que los artículos de la escala son representativos de algún universo definido, implica que el juicio de la gente esté familiarizado con el propósito del cuestionario, los mismos determinan, la correspondencia entre el dominio del contenido y los artículos de la medición. La validez de criterio establece que es válido el instrumento comparado con algún criterio externo y la validez del constructo, relaciona al grado que una medición se corresponde consistentemente con otras.

Entre los aspectos que pueden afectar la validez de un instrumento está la improvisación, la utilización de instrumentos desarrollados en otros países y que no han sido validados en contexto que se estudia, o que sea inadecuado a las personas a las que se les ha aplicado y que sea muy extenso [8-9].

\section{La confiabilidad o fiabilidad de un instrumento [10-11]}

En el presente caso fue utilizado el método de estimación de alfa de Cronbach, la que indica la profundidad con que los puntos del cuestionario están interrelacionados. La estimación de Cronbach de la fiabilidad se basa en la matriz de correlación de los ítemes donde se calculan los coeficientes de correlación de Pearson entre todos los ítemes. Para los cálculos se utilizaron los modelos que se describen a continuación.

$$
\begin{gathered}
\bar{p}=\frac{\sum p}{N P} \\
\alpha=\frac{N * \bar{p}}{1+p *(\bar{N}-1)}
\end{gathered}
$$

Donde:

$\bar{p}$ : Promedio de las correlaciones.

$N P$ : El número de correlaciones no repetidas.
$N$ : Tamaño de la muestra.

$\alpha$ : Coeficiente Alfa de Cronbach.

La mayoría de los autores coinciden en tomar como valor de $\alpha$ de Cronbach aceptable cuando se encuentra entre 0,70 y 0,80 , bueno entre $81 \%$ y 90\% y excelente superior al $90 \%$ [11].

\section{Selección de grupo a encuestar}

Para la selección del grupo de especialistas se tomó como base a graduados del nivel superior, con experiencia en el centro (al menos 10 años) y en las ramas de mantenimiento y producción, quedando integrado de la siguiente manera:

- Un jefe de sección, ingeniero mecánico, con 20 años de experiencia en el trabajo de mantenimiento y fundador del centro.

- Un especialista en diagnóstico, ingeniero mecánico, con 15 años de experiencia en el área de mantenimiento.

- Dos ingenieros mecánicos, con más de 10 años de experiencia en la actividad.

- Un jefe de planta, ingeniero químico, tecnólogo, con 12 años de experiencia en la actividad.

\section{Método de análisis de la encuesta}

En este caso se utilizó el método desarrollado por Rensis Likert o método de Likert [7], el que consiste en un conjunto de ítemes presentados en forma de afirmaciones o juicios, ante los cuales se mide la reacción de los sujetos que se le administra el instrumento.

Las puntaciones en la escala de Likert se obtienen sumando los valores obtenidos respecto a cada ítem, es por esto que se denomina escala aditiva. Una puntuación se considera alta o baja según la suma total de afirmaciones con respecto a la suma total de todas, también se califica el promedio obtenido en la escala mediante la fórmula PT/NT, donde PT es la puntuación total de la escala y NT es el número total de afirmaciones. Este método es estrictamente ordinal pero es común que se le trabaje como si fuera de intervalo. Este es el método fundamental que servirá para evaluar las encuestas.

Para comprobar la potencialidad de la herramienta se seleccionó como objeto de estudio para su implementación una planta típica, de las de mayor producción de bioproductos en Cuba. 


\section{RESULTADOS}

\section{Diseño del instrumento}

El instrumento o encuesta quedó estructurada de la siguiente manera:

Variable de salida o rendimiento: Estado de la gestión de mantenimiento en plantas de bioproductos.

Los elementos constitutivos se muestran en la Tabla 1.

Tabla 1. Elementos o variables constitutivas de la encuesta.

\begin{tabular}{|l|c|c|}
\hline \multicolumn{1}{|c|}{ Indicadores } & $\begin{array}{c}\text { Cantidad } \\
\text { de } \\
\text { dimensiones }\end{array}$ & $\begin{array}{c}\text { Cantidad } \\
\text { de } \\
\text { ítemes }\end{array}$ \\
\hline Estructura organizativa & 3 & 11 \\
\hline Servicios de mantenimiento & 5 & 19 \\
\hline Recursos humanos & 4 & 8 \\
\hline Producción & 1 & 2 \\
\hline Economía & 3 & 11 \\
\hline Logística & 1 & 7 \\
\hline Equipamiento & 8 & 13 \\
\hline Estrategias & 3 & 7 \\
\hline
\end{tabular}

A partir de los resultados que se muestran en la Tabla 1 se puede resumir que el instrumento quedó estructurado con un total de 8 indicadores, 28 dimensiones y 78 ítemes.

Las dimensiones e ítemes se especifican en la bibliografía citada [9], un ejemplo de la misma es:

Indicador: Estructura organizativa.

Dimensión: Nivel del mantenimiento dentro de la organización.

- Ítem: El área de mantenimiento se encuentra al mismo nivel en la toma de decisiones con respecto a las áreas de producción o de administración.

- Ítem: El área de mantenimiento tiene poder de decisión desde el inicio del proceso inversionista.

\section{Resultados de la encuesta}

Para el análisis de la validez del instrumento se tomó como base los criterios vertidos por el grupo de especialista de muestreo, concluyéndose que el instrumento mide el objeto de estudio, los ítemes dan respuesta al diseño de indicadores y dimensiones que se plantearon para medir la gestión del mantenimiento, en su confección se incluyeron criterios y observaciones de tres especialista en la materia de evaluación, por lo que no fue fruto de la improvisación ni de la traducción de una encuesta similar producida en el extranjero o en otra institución, los encuestados la entendieron vertiendo sus criterios a uno de los autores del presente trabajo que fungió como moderador.

\section{Cálculo de la confiabilidad de la encuesta}

En las Tablas 2 y 3 se muestran los resultados de los cálculos del $\alpha$ de Cronbach para cada Modelo, los valores de cada indicador, dimensión y el valor de la confiabilidad general del instrumento. Como se puede observar, no todas las dimensiones presentan resultados en las matrices debido a que no existe diferencia significativa entre los resultados recogidos en las encuestas, se señalan por $\mathrm{n} / \mathrm{d}$ o cuando solo la dimensión es valorada por un solo ítemes, se señala por $1 / \mathrm{IT}$

Del análisis de los resultados que se muestran en las Tablas 2 y 3 se puede observar que se obtiene para el Modelo I el $\alpha$ de Cronbach con un valor de $88 \%$ declarado como bueno y para el Modelo II un valor de $93 \%$ que se considera excelente. De igual forma se obtiene un valor total de la encuesta de $\mathbf{9 0 , 8 \%}$ declarado como excelente.

\section{Caso de estudio}

En la Figura 1 se muestra un gráfico resumen del comportamiento de los indicadores analizados contra los valores característicos de la escala de Likert.

A partir de criterios de los especialistas y la confiabilidad de la herramienta se propone:

Por debajo de 2,8 se tomará como un valor malo y la necesidad de implementar las estrategias que den respuestas a la solución de los problemas analizados. Entre 2,81 y 3,4 es regular el estado y se corregirán las estrategias con el fin de solucionar los problemas detectados. Mayor de 3,41 se debe detectar la existencia de algún elemento que sea factible su corrección para lograr mantener el buen funcionamiento de la organización. Se tomó 3 como valor central.

Se obtuvo que de forma general el estado de la gestión de mantenimiento de esta planta es mala $(2,06)$, por 
Tabla 2. Resultados del cálculo de los $\alpha$ de Cronbach, para el Modelo I.

\begin{tabular}{|c|c|c|}
\hline $\mathbf{N}^{0}$ & Dimensión & $\begin{array}{c}\alpha \text { de } \\
\text { Cronbach }\end{array}$ \\
\hline \multicolumn{3}{|c|}{ Modelo I } \\
\hline I1 & \multicolumn{2}{|l|}{ Estructura organizativa } \\
\hline D1 & $\begin{array}{l}\text { Nivel del mantenimiento dentro de } \\
\text { la organización. }\end{array}$ & $\mathrm{n} / \mathrm{d}$ \\
\hline D2 & Comunicación entre departamentos & $89 \%$ \\
\hline D3 & Gestión de la información. & $\mathrm{n} / \mathrm{d}$ \\
\hline \multicolumn{2}{|r|}{ Total del indicador I1 } & $89 \%$ \\
\hline $\mathbf{I 2}$ & \multicolumn{2}{|l|}{ Servicio de mantenimiento } \\
\hline D4 & Gestión de órdenes de trabajo. & $94 \%$ \\
\hline D5 & Eficiencia del área de mantenimiento & $91 \%$ \\
\hline D6 & Calidad del servicio de mantenimiento. & $91 \%$ \\
\hline D7 & Servicios de terceros. & $\mathrm{n} / \mathrm{d}$ \\
\hline D8 & $\begin{array}{l}\text { Gestión de herramientas y útiles de } \\
\text { mantenimiento. }\end{array}$ & $\mathrm{n} / \mathrm{d}$ \\
\hline \multicolumn{2}{|r|}{ Total del indicador $\mathbf{I} 2$} & $92 \%$ \\
\hline I3 & \multicolumn{2}{|l|}{ Recursos Humanos } \\
\hline D9 & $\begin{array}{l}\text { Fuerza laboral destinada al mante- } \\
\text { nimiento }\end{array}$ & $82 \%$ \\
\hline D10 & Certificación del personal & $1 / \mathrm{IT}$ \\
\hline D11 & Superación profesional individual & $1 / \mathrm{IT}$ \\
\hline D12 & $\begin{array}{l}\text { Disponibilidad de fuerza laborar de } \\
\text { mantenimiento }\end{array}$ & $100 \%$ \\
\hline \multicolumn{2}{|r|}{ Total del indicador I3 } & $91 \%$ \\
\hline I4 & \multicolumn{2}{|l|}{ Producción u operaciones } \\
\hline D13 & $\begin{array}{l}\text { Aprovechamiento de la maquinaria } \\
\text { productiva }\end{array}$ & $72 \%$ \\
\hline \multicolumn{2}{|r|}{ Total del indicador I4 } & $72 \%$ \\
\hline I5 & \multicolumn{2}{|l|}{ Economía } \\
\hline D14 & $\begin{array}{l}\text { Definición de los presupuestos de } \\
\text { mantenimiento }\end{array}$ & $97 \%$ \\
\hline D15 & Control de costo de mano de obra & $93 \%$ \\
\hline D16 & Control de costos de materiales & $95 \%$ \\
\hline \multicolumn{2}{|r|}{ Total del indicador I5 } & $95 \%$ \\
\hline I6 & \multicolumn{2}{|l|}{ Logística } \\
\hline D17 & Gestión de almacenes & $93 \%$ \\
\hline & Total del indicador I6 & $93 \%$ \\
\hline & Total del Modelo I & $88 \%$ \\
\hline
\end{tabular}

Tabla 3. Resultados del cálculo del $\alpha$ de Cronbach, para el Modelo II y el valor total del instrumento.

\begin{tabular}{|c|c|c|}
\hline $\mathbf{N}^{\circ}$ & Dimensión & $\begin{array}{c}\alpha \text { de } \\
\text { Cronbach }\end{array}$ \\
\hline \multicolumn{3}{|c|}{ Modelo II } \\
\hline I7 & Equipamiento & \\
\hline D18 & Gestión de activos & $87 \%$ \\
\hline D19 & Tiempo promedio entre fallos & $1 / \mathrm{IT}$ \\
\hline D20 & Tiempo promedio para fallo & $1 / \mathrm{IT}$ \\
\hline D21 & Disponibilidad técnica & $1 / \mathrm{IT}$ \\
\hline D22 & Tiempo promedio de reparación & $1 / \mathrm{IT}$ \\
\hline D23 & $\begin{array}{l}\text { Costo de mantenimiento por el } \\
\text { valor de reposición }\end{array}$ & $1 / \mathrm{IT}$ \\
\hline D24 & $\begin{array}{l}\text { Costo de mantenimiento por } \\
\text { facturación }\end{array}$ & $80 \%$ \\
\hline D25 & Gestión de históricos & $100 \%$ \\
\hline \multicolumn{2}{|r|}{ Total del indicador I7 } & $89 \%$ \\
\hline I8 & Estrategias de mantenimiento & \\
\hline D26 & Mantenimiento productivo total. & $1 / \mathrm{IT}$ \\
\hline D27 & Planificación & $1 / \mathrm{IT}$ \\
\hline D28 & Programación & $97 \%$ \\
\hline & Total del indicador I8 & $97 \%$ \\
\hline & Total del Modelo II & $93 \%$ \\
\hline & Total de la Encuesta & $90,8 \%$ \\
\hline
\end{tabular}

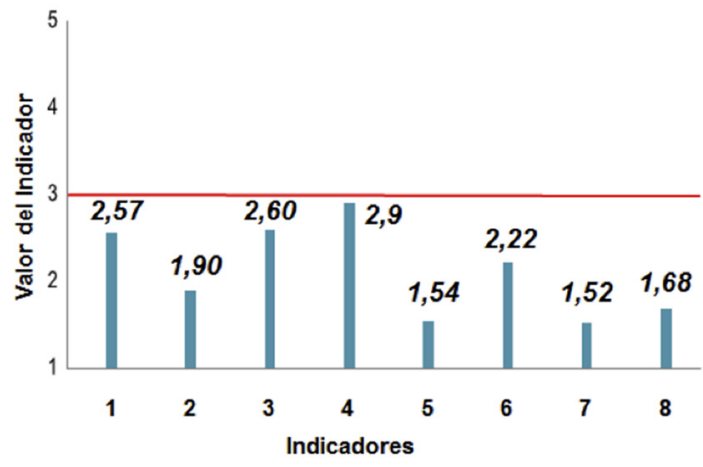

Figura 1. Gráfico de comportamiento de los indicadores contra su valor obtenido. 
lo que se recomienda un análisis profundo por la dirección de la entidad en atención a los problemas planteados para tomar decisiones que logren que mantenimiento ocupe su lugar y cumpla con sus funciones. A continuación se detallan algunas de las principales problemáticas detectadas para cada uno de los indicadores.

I.1. Estructura organizativa: El área de mantenimiento no guarda el mismo nivel en la toma de decisiones que otras áreas de la entidad. La información que se recibe de otros departamentos no llega con la calidad y rapidez requerida por esta área, no contándose con sistemas informáticos para su gestión.

I.2. Servicios de mantenimiento: no se cuenta con el documento órdenes de trabajo. No se registran sistemáticamente las causas reportadas por los clientes.

I.3. Recursos Humanos: El área de mantenimiento no cuenta con la plantilla adecuada para prestar un servicio de calidad, no se realizan superaciones ni adiestramientos a los trabajadores, no se realizan análisis del tiempo empleado en actividades reactivas con respecto a las proactivas.

I.4. Producción u operaciones: Existe relación entre mantenimiento y producción, mantenimiento ajusta sus tareas a ejecutar teniendo en cuenta el tiempo de explotación planificado.

I.5. Economía: La definición de los presupuestos para mantenimiento son realizados por directivos de la entidad sin tener en cuenta criterios de los especialistas de mantenimiento ni se realizan análisis históricos de actividades similares ejecutadas con anterioridad.

I.6. Logística: No se planifican los trabajos atendiendo a recursos existentes, no existen sistemas informáticos que realicen la gestión de almacenes.

I.7. Equipamiento: Es de mal la gestión de los activos. No se realizan análisis de costos de reparación ni de facturación. No se controlan sistemáticamente los tiempos promedios para reparación. No se analizan los históricos.

I.8. Estrategias: Se vincula personal de producción a labores de mantenimiento. No se realizan inspecciones para monitorear sistemáticamente parámetros de estado a los activos y ejecutar acciones concretas, no se planifican acciones de mantenimiento según el contexto operacional de los equipos.
Con la implementación de esta herramienta a un caso de estudio se diagnosticó el estado de la función mantenimiento en una planta demostrándose además la pertinencia de la herramienta para el campo objeto de estudio.

\section{CONCLUSIONES}

Se obtuvieron los indicadores, dimensiones e ítemes que sirvieron para evaluar la variable de salida o rendimiento gestión de mantenimiento, con un total de ocho indicadores, 28 dimensiones y 78 ítemes.

El instrumento diseñado permite cuantificar la variable de salida, gestión de mantenimiento en plantas de bioproductos, el mismo satisface los requerimientos de validez verificado según el criterio del constructo y una confiabilidad del $90,8 \%$, declarado excelente.

Con la aplicación de la herramienta a un caso de estudio se pudo estimar el estado de la gestión de mantenimiento en una planta de bioproductos la cual arrojó un estado insatisfactorio.

\section{REFERENCIAS}

[1] H. Acosta. "Propuesta de documento guía para el diagnóstico y evaluación de la función mantenimiento en el sector hotelero y hospitalario". Tesis en opción al grado de máster en ingeniería mecánica. Centro de estudios en ingeniería del mantenimiento. Instituto Superior Politécnico José A. Echeverría. La Habana, Cuba. 2007. URL: http://biblioteca.cujae.edu.cu:81/TesisCujae/ busqueda/acosta hector/find.asp Fecha de consulta: 20 de julio de 2015.

[2] E. Falcón Oconor, M. Rivera Nelson. "Efecto de la inoculación de hongos micorrizógenos sobre la producción de posturas forestales en dos tipos de suelos". Revista cultivos tropicales. Vol. $34 \mathrm{~N}^{\circ}$ 3. Julio - septiembre 2013. ISSN 0258-5936. URL: http:// scielo.sld.cu/scielo.php?pid=S0258$59362013000300005 \&$ script=sci_arttext. Fecha de consulta 22 de abril 2016.

[3] A. Díaz y A. Castillo. "Propuesta de un modelo para el análisis de criticidad en plantas de productos biológicos". Revista Ingeniería Mecánica Vol. 15 No 1, pp. 34-43. 
Enero-abril, 2012 ISSN: 1815-5944 URL: http://www.ingenieriamecanica.cujae.edu. cu. Fecha de consulta: 10 de mayo de 2015. C. Alveiro Montoya. "El Balanced Scorecard como herramienta de evaluación en la Gestión administrativa". Visión del futuro. Vol. 15 $\mathrm{N}^{\circ}$ 2, pp. 1-25. Diciembre 2011. ISNN: 1668-8708. URL: http://revistacientifica.fce. unam.edu.ar/index.php?option=com_conten $\mathrm{t} \&$ view $=$ article $\& i d=251 \&$ Itemid=61. Fecha de consulta: 12 de enero de 2015.

[5] E.J. Vásquez. "Modelo para auditar la gestión de mantenimiento de PDVSA caso: refinería SAN ROQUE". Revista Mantenimiento en Latino América. Vol. $4 \mathrm{~N}^{\circ}$ 3, pp. 10-20. Mayo-junio 2012. ISSN: 2357-6840.

[6] L. Ruiz. "Propuesta de mejora del mantenimiento en los hornos de fundición de la UEB Cubana de Bronce". Tesis en opción al grado de máster en ingeniería mecánica. Centro de estudios en ingeniería del mantenimiento. Instituto Superior Politécnico José A. Echeverría. La Habana, Cuba. 2015. URL: http://biblioteca.cujae. edu.cu:81/tesispdf/Maestria/Maestria1959. pdf. Fecha de consulta: 25 de abril 2016.

[7] B.E. Hayes. "Como medir la satisfacción de cliente". Ediciones Gestión 2000, pp. 103-180. Edición II. Barcelona, España. 1995. ISBN: 848088696X. URL: http:// www.agapea.com/libros/Como-medir-lasatisfaccion-del-cliente-9788480886963-i. htm. Fecha de consulta: 15 de marzo 2015.
[8] M. Valle Flórez. "Dimensiones de análisis de la investigación en comunicación". Revista Luciérnaga Audiovisual. Politécnico Colombiano Jaime Isaza Cadavid. Edición 5.2011. ISSN 2027 - 1557 URL: http:// politecnicojic.edu.co/luciernaga5/pdf/. Fecha de consulta: 21 de abril 2016.

[9] R. Hernández Sampieri. "Metodología de la Investigación". Editorial Mc Graw Hill. Cuarta Edición. D.F., México. pp. 208-300. 2012. ISBN: 970-10-5753-8. URL: https://competenciashg.files.wordpress. com/2012/10/sampieri-et-al-metodologia-dela-investigacion-4ta-edicion-sampieri-2006_ ocr.pdf. Fecha de consulta: 18 de abril 2015.

[10] J.A. Bojórquez Molina, y Col at. "Utilización del alfa de Cronbach para validar la confiabilidad de un instrumento de medición de satisfacción del estudiante en el uso del software Minitab". Eleventh LACCEI Latin American and Caribbean Conference for Engineering and Technology (LACCEI'2013)" Cancún, México. 2013. URL: http://www.laccei.org/LACCEI2013Cancun/. Fecha de consulta: 18 de abril 2015.

[11] S.F. Soler Cárdenas, L. Soler Pons "Usos del coeficiente alfa de Cronbach en el análisis de instrumentos escritos": Revista Médica Electrónica. vol. $34 \mathrm{~N}^{\circ}$ 1. pp. 1-6. Matanzas. ene.- feb. 2012. ISSN 1684-1824. URL. http:// scielo.sld.cu/scielo.php?script=sci_arttext\& pid=S1684-18242012000100001. Fecha de consulta: 15 de mayo 2015. 